\title{
POP-UP CONCRETE CONSTRUCTIONS: FORMING FABRIC REINFORCED CONCRETE SHEETS
}

\author{
A. N. VAZQUEZ \& W. JABI \\ Welsh School of Architecture, Cardiff University.
}

\begin{abstract}
New technologies and fabrication tools urge us to explore new materials and their potential for integration in architectural construction. One such material, Concrete Canvas, is explored in this paper for its hybrid characteristics that blend fabric and thin-shell tectonics. The potential of Concrete Canvas lies in its ability to modify itself from a flexible fabric that when activated with water becomes a rigid concrete structure. Combined with a digitally controlled workflow of on-site cutting and an iterative material feedback loop, the process can serve as a radical alternative to current concrete formwork fabrication techniques. This paper outlines a prototypical design process that combines a phase-changing material, physical computer simulations, robotic fabrication and scanning technologies on a feedback loop between the digital and the physical that allow for customized, free-form, on-site concrete structures to pop-up without the need of a complex formwork. In this process the architect sets the various parameters based on fabrication techniques and material properties and adjusts them iteratively in the physical and digital model during the 'popping-up' process until a balance between material properties, technical requirements and aesthetics is reached, exploring new potentials on digital fabrication processes. The paper outlines the proposed workflow including iterative experiments with robotic cutting of flat patterns, their 'popping-up' into 3D concrete shells, and material phase transitions during its forming process. The established feedback loop consisting of geometry scanning, parametric perforation pattern control, computational analysis and simulation, and robotic fabrication is described in detail. The paper concludes by exploring the potential of this process to enable a dialogue between digital architecture and the process of materialization and discusses the implications of this approach in relation to architectural design and fabrication workflows.
\end{abstract}

Keywords: adaptable, digital fabrication, form-finding, pop-up concrete, robotic fabrication, transformable structures.

\section{INTRODUCTION}

In 1968, David Pye made a well-known distinction between the workmanship of risk defined by the process of making and the world of the materials and the workmanship of certainty defined by the world of manufacturing and mass production [1]. He further clarified that the risks are not only in the materials but also in any kind of technique where there is uncertainty with regards to the results because they depend on a variety of external factors that put the result continuously at risk during the process of making [1]. This differentiating relationship becomes particularly interesting when adopting relatively new processes within traditional manufacturing tools where the results are neither completely predictable nor controlled. In that scenario, the digital model no longer prescribes a form into the material but gives the form the capacity to emerge by embedding the necessary information in the material itself. This process is not unique to digital fabrication techniques. Buckminster Fuller, Frei Otto and other form finders of the 1970s were interested in the potential of what materials can be. They developed a hands-off approach to design based on adaptable and flexible solutions and demonstrated the importance of establishing a relationship between performance and material integrity from the early stages of design [2].

Digital form finding methods are optimized to create forms according to structural behaviours and the flow of forces within their boundaries. Despite an increase in their development 
and use across architectural practice, they are not optimized to include material behaviours and properties which are affected by a large amount of variables that can be computationally intensive. This has resulted in form taking precedence over material even though it is material through which forces flow [3]. This disconnection means that geometries have to undergo an intensive process of rationalization and 'making buildable' where friction occurs between the material, the fabrication processes and the final form.

A different approach to manufacturing and to design is required to achieve individualized fabrication on a large scale and create the desired forms of contemporary architectural language. The arrival of new tools and techniques has always posed a challenge for construction and architecture and required a new way of thinking and doing [4]. Digital fabrication tools are allowing architects to eliminate the drawing and the model as intermediaries between design and fabrication and work directly with the materials manipulating their logic and understanding them similarly to the way painters and sculptors understood and worked directly with their artifacts [5]. While these tools have allowed architects to be in direct contact with their medium and to explore processes of fabrication that negotiate the relationships between control and risk similar to craft processes, they presume a unidirectional and disjointed process that relies on off-site prefabrication production methods. In this paper, the use of digital fabrication tools and material agency is put forward not as a unidirectional process from 'digital input' to 'physical output', but as a continuous and iterative feedback loop between both.

\section{POP-UP CONCRETE}

Concrete has been used for a long time and the interest in building concrete free-forms has gained relevance in the last decade, which has encouraged a large amount of robotic and non-robotic research in flexible formwork systems [6]. It is clear that when building concrete free-forms, one crucial decision is the choice of formwork to guarantee its quality and financial feasibility [7]. Despite the increased interest in free-form concrete and the vast amount of research on flexible formwork, most digital forms are still built using traditional formwork and methods. The problem lays in the fact that construction processes still rely on a unidirectional workflow from 'digital design' to 'physical production'. This means that designs have to go through a lengthy rationalization process where friction between form, structure and material occurs. An integrated design workflow is researched and presented in this paper that

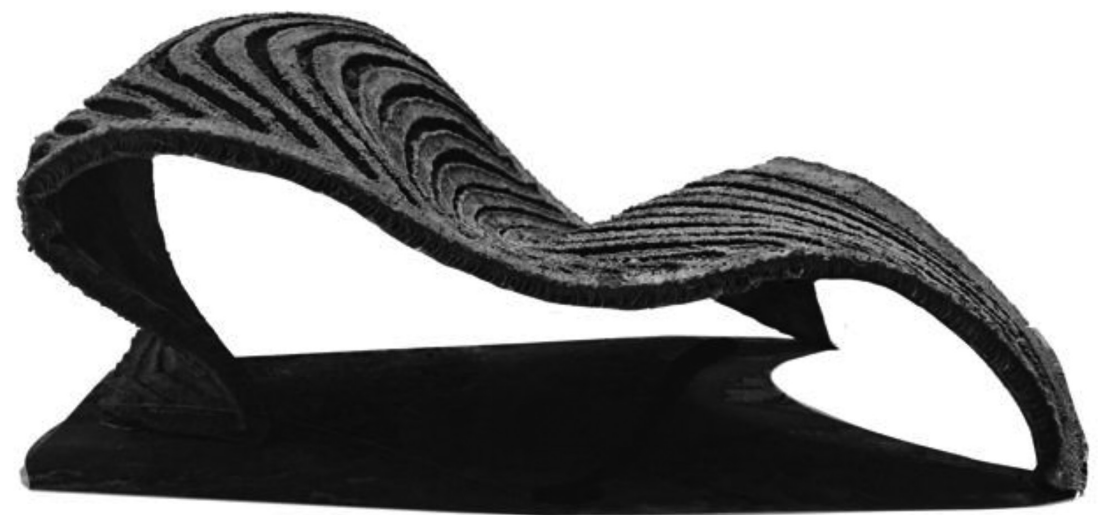

Figure 1: Popped-up concrete canvas prototype. 
integrates design and building through the use of new material technologies and digital fabrication tools.

Technical devices and digital fabrication tools allow for new practices and are capable of opening new understandings of matter, new ways of organizing, and new complex and irregular relationships that expand material processes to create new non-linear workflows and can lead to a new language characteristic of the robotic era in architecture. Using a new material technology within a pop-up process, based on patterns that embed the shape into the material rather than prescribe it, requires an experimental approach as the material exhibits probable but not certain behaviour. Thus, a new path, based on feedback loops, is proposed toward the design of curved, thin, flexible structures in concrete without the need for complex formwork that would be otherwise required [8]. Our shaping system allows for complex curves to be created through a combination of the concrete sheet material and the embedded pattern.

\section{PRECEDENT ANALYSIS}

In the context of robotic fabrication of concrete, projects to date have been divided into four major areas of exploration, each with a unique set of limitations:

1. Concrete 3D printing: Over 10 years ago researchers at the Universities of Southern California [9] and Loughborough [10] started to investigate the potential of extruding concrete for printing buildings. Both attempts used an extrusion head mounted on a gantry crane to deposit horizontal layers of concrete. However, limitations exist regarding the scalability of the gantry, the hydration process, the loading capacity, the adhesion of the different layers, and the integration of reinforcement.

2. Dynamic Formwork: 'Smart Dynamic Casting' [11] focuses on the vertical extrusion of concrete columns, using sensors and a feedback loop to monitor and control the hydrating of the concrete. This information is then used to determine the slip velocity, tackling the problems of previous 3D printing methods. The careful calibration of sensor feedback with the spatial movement allows for a high level of control over the formation process.

3. Mould-based formwork, flexible and rigid: Tailor Crete developed a digitally controlled, recyclable, flexible, wax mould system that is produced off-site, which is then brought on-site and inserted into standard formwork systems to produce complex concrete structures [12]. Conversely, the 'UNIKAbeton' prototype showed the possibilities for complex concrete construction using digitally fabricated rigid EPS blocks [13].

4. Leave-In formwork: The mediated matter group at the MIT [14] and the project 'Mesh Mould' at the ETH [15] explore techniques where the robot 3D prints permanent formwork, which doubles in function as thermal insulation in the former and as reinforcement in the latter.

\section{METHODOLOGY}

Pop-up is a technique that transforms planar materials into 3D forms. Research in pop-up as a construction system in architecture and other fields remains relatively unexplored. Researchers in nano-materials have only recently started to look at the potential of pop-up as a manufacturing technique and as a simpler route to achieve 3D frameworks by buckling planar structures, allowing them to create complex shapes using a variety of materials such as silicon and semiconductors [16]. There is also on-going research using a pop-up system on modified crystals for implantable devices that can be triggered to morph once inside the 

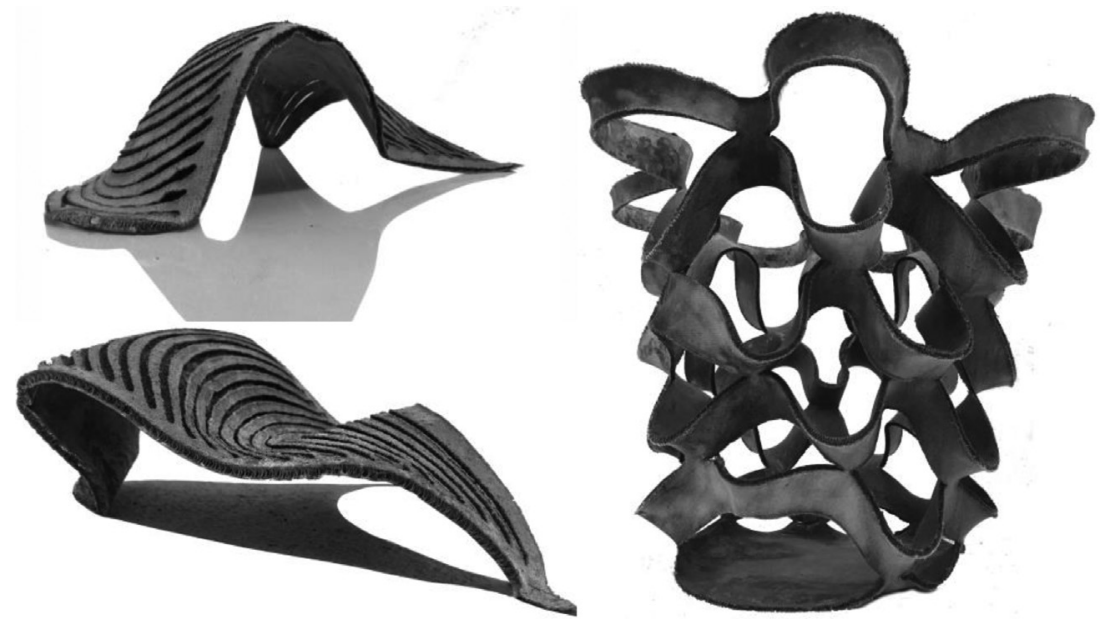

Figure 2: A vocabulary of pop-up structures is starting to develop, result of the design process.

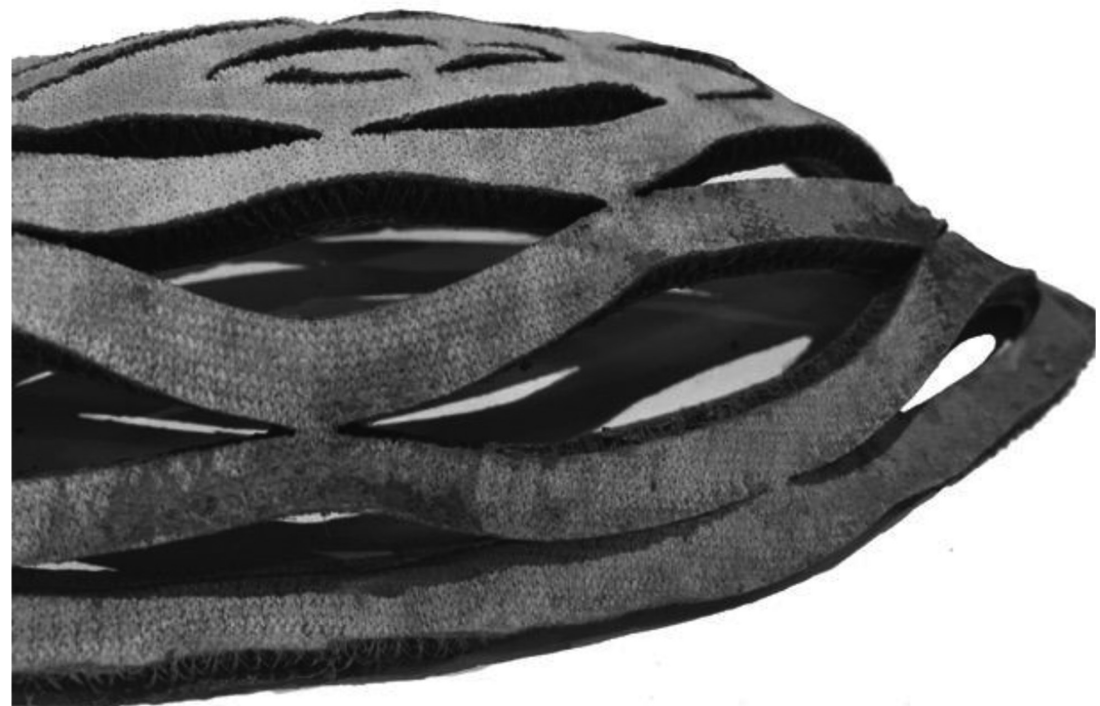

Figure 3: Popped-up shell prototype.

body [17]. The formation of pop-up structures is not random but caused by set boundary conditions of the embedded cut and joint pattern and follows precise physical principles.

Pop-up geometries are marked with a number of challenges or 'risks' that are not common to geometries that are first digitally modelled and then fabricated in a traditionally unidirectional process. A vocabulary of geometries is starting to develop from these initial experiments with certain characteristics and material properties through which we explore the potential of popped-up concrete geometries. The feedback mechanism allows us to refine the patterning of a construction material like concrete and its popping up process, exert more control over the system and explore opportunities for further improvements of the physical prototypes that ultimately will bring the work closer to full scale applications. 

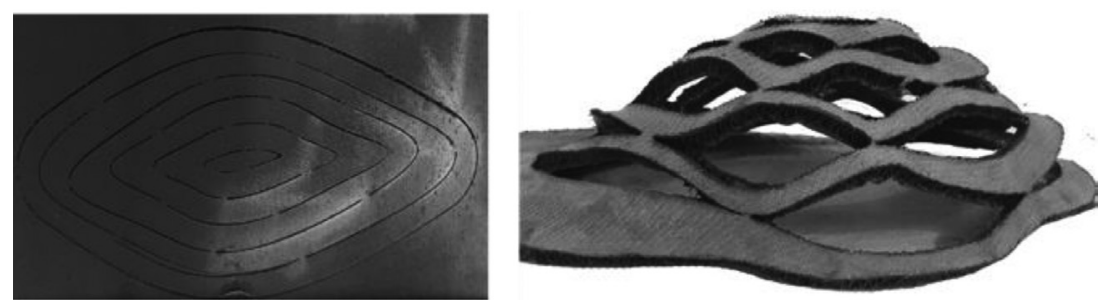

Figure 4: Left: 2D pattern laser cut in concrete canvas. Right: Popped-up concrete canvas shell prototype.

These concrete geometries rely on a system based on 2D cutting patterns performed in 'concrete canvas', described below, that transforms into a 3D shape by buckling on site using inflation to create a surface. The concrete canvas cures with the addition of water to become structurally rigid after an initial period of 5 hours and becomes fully set after 24 hours. Concrete shaping is possible as long as the concrete is in its wet state; this curing period or 'transition' phase of the concrete opens possibilities for new shaping strategies where the form of the 3D object is transformed. Digitally, 3D shapes can be collapsed into 2D cutting patterns to be popped back up into 3D surfaces. The design is not finalized until the material hardens giving various opportunities for interaction between the architect and the material and thus making fabrication and interactive process of creation.

\section{PHYSICAL FABRICATION}

\subsection{Concrete canvas}

New materials provide an opportunity for designers to create new typologies [18]. Material developments and higher strength concrete have been used to explore 3D complex concrete shapes that pop-up from flat 2D patterns. Concrete is not traditionally a flat sheet material. However, fabric impregnated concrete, a new hybrid material technology, combines the compressive strength of concrete and the tensile strength of fabric. This seemingly contradictory characteristic allows for a more intuitive design workflow that can lead to a flexible and adaptive design process. Through prototype testing it became clear that a feedback step is needed within the process to address the possibilities and uncertainties presented by the material when used in novel ways.

Concrete canvas allows easy deployment and rapid construction of thin concrete shells (http://www.concretecanvas.com) as it only requires air and water for construction. Shelter structures up to $50 \mathrm{sqm}$ have been built using this material. It consists of two flexible membranes on each exterior surface with a 3D fibre matrix impregnated with cement. The top
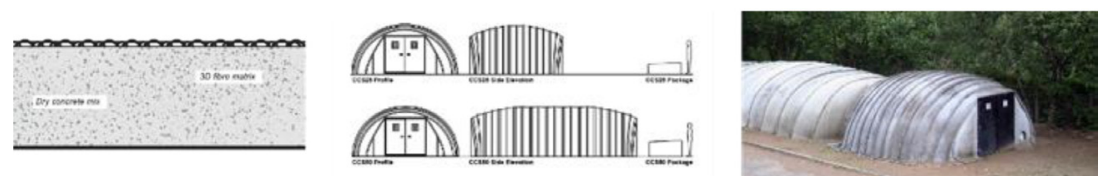

Figure 5: Left: Concrete canvas section. Middle: Typical deployment sequence. Right: Shelter structure. Source www.concretecanvas.com. 
layer is a fibrous surface that can be hydrated while the back membrane is made of waterproof fire-resistant PVC. The cement-based composite fabric uses inflation to create its surfaces that are optimized for compressive loading. When hydrated after 24 hours, the membranes harden forming a thin, robust and lightweight concrete structure. Concrete canvas comes in different thicknesses $(5,8$, and $13 \mathrm{~mm})$. The experiments described in this paper use the $5 \mathrm{~mm}$ variety.

\subsection{Robotic tooling}

A set of key variables was identified for the design of the robot tool such as: the turning radius of the cuts, the depth of the sandwiched material and the robot's cutting speed. A laser cutter was used initially but the additional installation requirements made it unsuitable for on-site applications. Circular diamond saws were also tested but the speed at which they needed to rotate caused concrete powder to eject and weakened the overall structure. A solution using a $45 \mathrm{~mm}$ diameter sharp circular blade was selected because it allowed efficient cutting, smaller turning radii, and lower rotational speeds. Enough depth is needed at the entry points so that it cuts all the way through the material using a single pass.

\subsection{Surface definition and tool path generation}

The process starts with the definition of a base surface. A control pattern of cuts and joints that will define the surface form is then applied. Four main criteria that define the final popped-up geometry are identified and parametrically controlled [19]:

1. The cutting pattern defines the relationship between the $2 \mathrm{D}$ pattern and the $3 \mathrm{D}$ volume. The cuts on a flat material need to be offset to achieve a concave geometry. The spacing between the cuts needs to consider the material behaviour and avoid extreme clustering
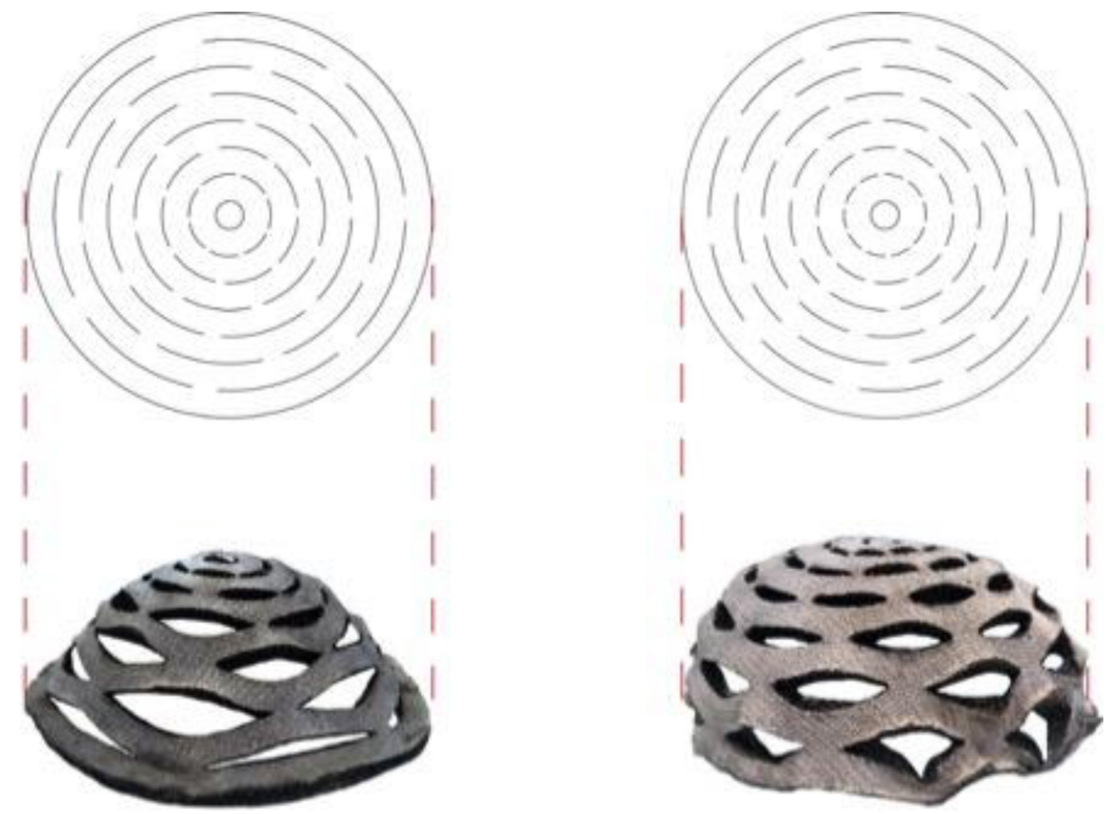

Figure 6 
that will result in long thin elements that can buckle given a very small distance from the edges. If the cuts are too far apart then the pop-up will be too shallow. A minimum section of $30 \mathrm{~mm}$ has been established for the $1.0 \times 1.0 \mathrm{~m}$ prototypes.

2. The joints between cuts affect the stability of the overall structure. The joints are the areas where there is no cut and are crucial for the popping of the unit. When joints are staggered, a more rigid structural system is achieved. Our experiments show that joints of 40-50 mm create rigid conditions. Joints smaller than that create flexible and semiflexible conditions.

3. Relaxation, form manipulation and inflation determine the final position and shape of the surface.

4. Pre-hydration and drying times affect both the structural rigidity of the surface as well as its elasticity. In our experiments, we tested different sequences of hydration and cutting to maintain the integrity of the final form and minimize concrete loss.

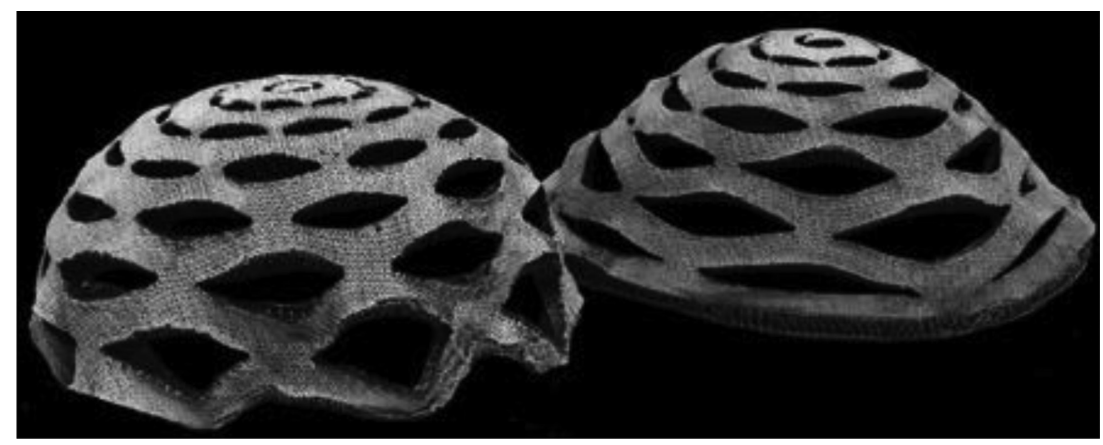

Figure 7: Changes to the cut and joint pattern, boundary conditions and relaxation constant.
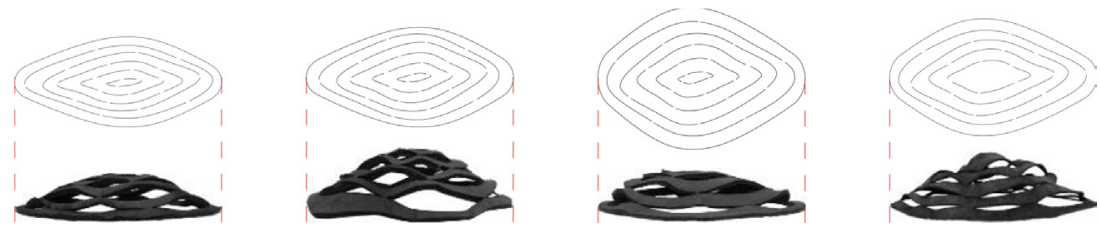

Figure 8: Comparison of geometric differences in the 3D pop-up surfaces, product of changes in boundary conditions and cutting patterns.
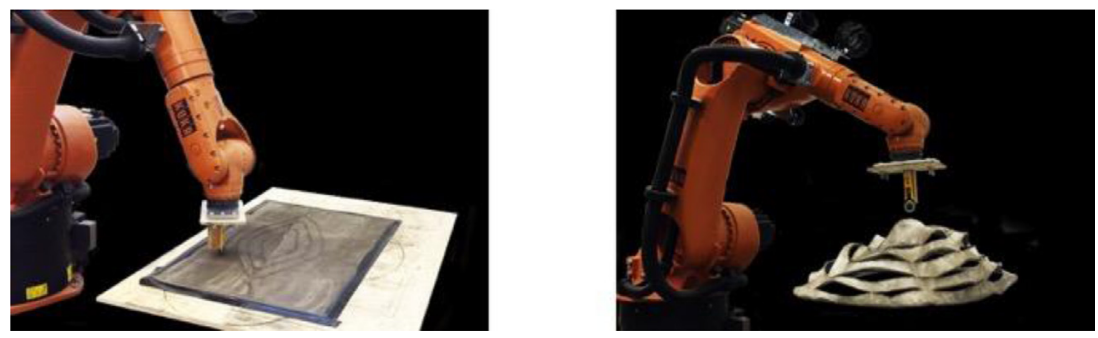

Figure 9: Left: Robot cutting pattern in concrete. Right: $1.0 \times 0.7 \times 0.7 \mathrm{~m}$ popped-up prototype. 
Once a pattern of cuts and joints is determined based on aesthetic and structural constraints, the curves need to be rationalized to maximize continuity. This ensures path continuity and decreases the possibility of singularities and out-of-reach positions for the robot. The end points of each curve are offset on the Z-axis for the robot to move vertically after each cut and allow for the joint areas.

\section{DIGITAL COMPUTATION AND SOFTWARE WORKFLOW}

The digital process is set with the aim to foresee materialization and control it during its forming. It required the customization and integration of different software platforms for material computing, physics solvers simulation and structural analysis. To achieve the initial goal of merging modelling, analysis and fabrication into a single process the form-found geometries need to be brought back into the digital world and a direct link needs to be created between the digital and the physical models.

After the initial surface with the joints and cut patterns is defined and modified it is exported to form-finding software based on particle spring systems. In this case Grasshopper and Maya nucleus solver were used to approximate the shape digitally. The Autodesk Maya $\mathrm{N}$-cloth delivers sufficiently accurate results in replicating the material performance and pop-up behaviour observed in the physical tests as it allows embedding and calibrating different physical constraints such as damping, strength, stiffness and density. Each pattern was established as a boundary condition and relaxed to find its resultant pop-up geometry within the pattern. Once the pop-ups are generated and evaluated the pattern is turned into tool paths using a custom-made robot communication platform for cutting and physical testing. Further research is being conducted to develop a workflow that integrates the robotic and physics simulation into a single platform, to enable a continuous workflow from design to realization of non-standard, material-driven fabrication processes.

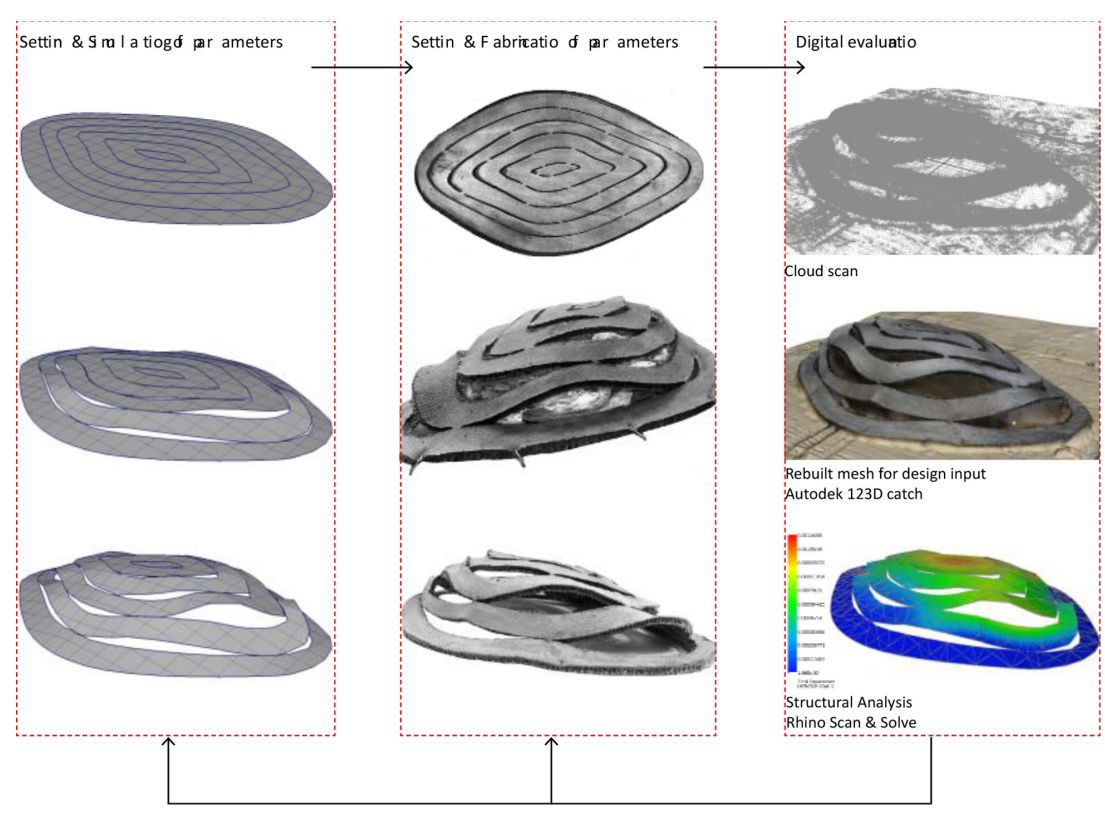

Figure 10: Diagram showing the workflow set out and digital-material feedback loop. 


\subsection{Feedback loops}

Utilizing a commercially available 3D scanning application, a strategy was evaluated in this research project for its potential in establishing the following iterative feedback loop: material deployment, automated inflation process, measurement of deformations in the physical geometry, calibration of the digital mesh, structural and aesthetic analysis of both, live manipulation of the inflated concrete structure and point-cloud 3D re-scanning.

In the implemented approach using Autodesk 123Dcatch and Photo Scan Pro the scanned information consists of a point cloud and a mesh that can be imported to the digital environment. This is then used to calibrate different parameters such as damping, strength, spring stiffness and density to approximate the digital and material behaviour. This allows the designer to quickly understand and evaluate the many factors that influence the process and to 'mould' the material. Feedback loops enrich the process as this information is taken to the following cut pattern. Enhancements or modifications to the cut pattern of the flexible sheet material are related to the whole process as the cut and joint strategy gives unique identifiable characteristics to the final rigid material. The iterative process allows us to integrate computational and material logic into the design with which we can predict and orchestrate sequential material behaviour. It negates the unidirectional flow from 'digital input' to 'physical output' that pervades current processes of digital fabrication.

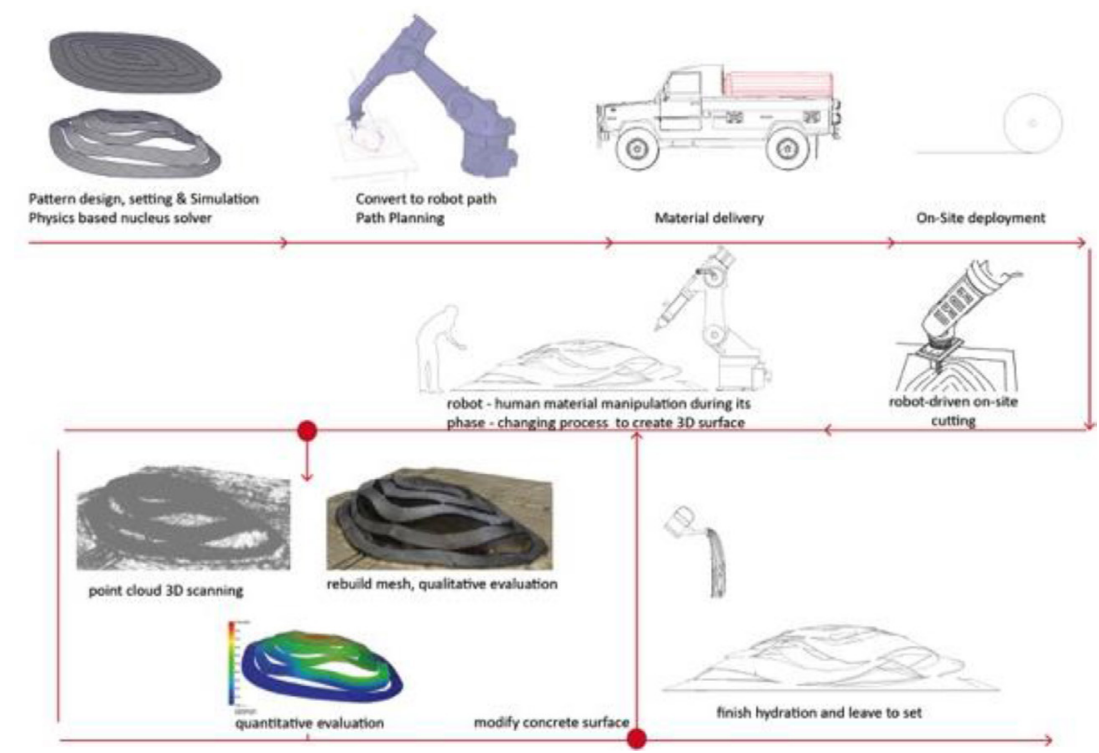

Figure 11: Path planning workflow and feedback loop.

\subsection{Analysis}

The uncertainties regarding the behaviour of the concrete canvas with the applied 'cuts and joints' pattern intertwined with the fact that the pattern can allow for material extension beyond its safe limits requires continuous analysis. Scale models were built and popped-up. Through scanning, the response of the model to the pattern was measured and its structural behaviour analysed and calibrated with the digital model. Modifying the control cut pattern 

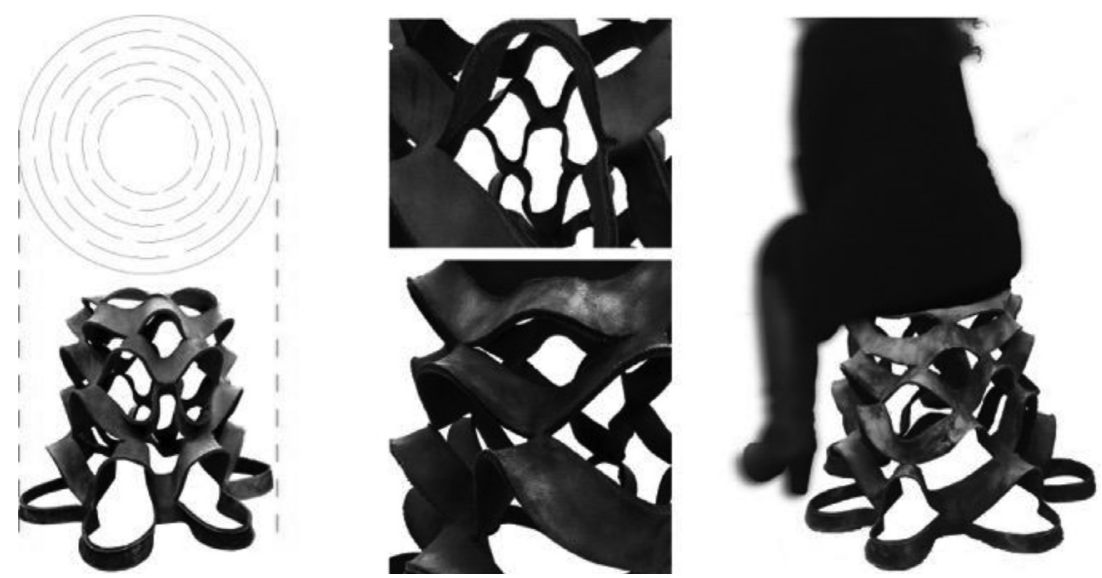

Figure 12: Left: Pattern and resultant pop-up geometry. Middle: Concrete details. Right: Live load testing to calibrate with structural assumptions.

gives different properties to the material. What was expected to be a homogeneous shell became flexible, semi-flexible and rigid. Rhinoceros Scan \& Solve was used to check the shapes qualitatively. This allowed a clearer image of the structural and material response to the cutting pattern.

\subsection{Simulation}

Iterative digital physics-based simulations were used to gain a deeper understanding of the relationship between the cut patterns and the final 3D form. The production of lowresolution meshes using particle-spring systems is an established practice for physics simulations. They provide the designer with an intuitive and qualitative knowledge during early design stages that can be augmented with structural and fabrication constraints through a feedback loop [20]. Calibrating a digital low resolution mesh with the high resolution material input from the scanning process allows the designer to work interactively with the geometry while enclosing all the important technical details such as singularity points, boundary and topological conditions, holes, clearances, etc. [21]. It also allows for an iterative quick evaluation of a range of options by adjusting key parameters that affect each realization [22].

\subsection{Flexibility}

The ability of the designer to intervene at any stage during the process is very important. During the inflation process through the feedback loop the designer has the flexibility to interrupt and change the flow of information. The resultant geometry can then be analysed for its structural and aesthetic characteristics while changing the parameters and consequently the geometry itself before hydration and curing.

\section{SCALABILITY}

Experiments so far have been limited in size by the maximum width of the fabric of 1,030 mm. To move into larger structures, concrete canvas geometries will have to be constructed 
assembling segments to complete the form before inflation and hydration. The fabric-like properties of the material enable the possibility of sewing various pieces together following a pattern as with traditional fabric formwork. This means that geometries that can be unrolled and cut out of fabric can be done using this technique. Concrete Canvas trademark shelters are built using this approach. To succeed at 1:1 scale using concrete impregnated fabric adjustments to the sequencing and construction planning are being explored while the fundamental concept remains feasible.

\section{HYBRID TECTONICS}

Additive, subtractive and formative processes are the three main accepted fabrication categories [23]. Embedding patterns in the concrete fabric is mainly a subtractive process in a homogeneous material. The distribution of cuts and joints gives areas of varying rigidity within the final form. This is the first step in introducing heterogeneous properties to the material. Based on the analysis of the 2D patterns and their 3D pop-up resultant geometry, further research is being done on ways to add material to reinforce specific areas during and after pop-up. A 3D printing step before popping-up can be an option to reinforce zones where more structural resistance is needed and that can be weak after pop-up. Introducing the possibility of adding material to the process gives the ability to tailor structural and material properties of an otherwise homogeneous material to improve efficiency and functionality in the final 3D geometry.

\section{CONCLUSIONS}

The implementations of pop-up structures that generate 3D surfaces out of 2D patterns clearly yielded an expanded domain for design exploration that can generate a new language for architecture in the robotic era. The generation of pop-up structures is not random but caused by set boundary conditions of the embedded cut and joint pattern and follows precise physical principles during its pop-up. Through the feedback loop and with defined boundary conditions the results can indirectly be controlled and emergent shapes be created by stopping the process at any point in time during the 'pop-up' phase of the concrete. 3D pop-up geometries can achieve a space-enclosing surface faster than 3D printed ones. A main challenge of this technique is that while the desired end $3 \mathrm{D}$ shape is known the pattern to produce it is not, an inverse situation to that of traditional construction methods [24]. Future work will be conducted to develop 2D patterns that pop-up into the desired 3D structure.

Initial experiments were concentrating on the development of pop-up strategies for industrially prefabricated products like 'Concrete Canvas'. However, this product is mainly used for infrastructure, roads and its structure and finish often doesn't allow the desired forming. For the next test scenarios, the authors want to include the design of the composite material itself, as it promises a huge impact on the formal results caused by the formation strategy.

At a design level the aim is to establish more intricate and larger patterns where two or more sheets are sewn together and their initial configuration responds to more complex geometries. The scanning of the physical geometry after being streamed to the digital simulation for analysis will be used to automate the control of the popping-up, in order to investigate viable inflating and interaction sequences that allow for closer relationships between designer, robot and material before implementing them in real time for a full-scale prototype. Through this exploration we anticipate the realization of complex concrete geometries responsive to embedded performance criteria. This research project points to a future where digital and material realities are constantly interacting within a continuous and iterative 
feedback loop and where digital fabrication tools are not only translators of predefined designs but enable a dialogue that brings the architect closer to the material.

\section{REFERENCES}

[1] Pye, D., The Nature and Art of Workmanship, Cambridge University Press: London, 1968.

[2] Oxman, N. \& Rosenberg, J.L., Material-based design computation: an inquiry into digital simulation of physical material properties as design generators. International Journal of Architectural Computing, 5(1), pp. 26-44, 2009. http://dx.doi.org/10.1260/147807707780912985

[3] Kotnik, T. \& Weinstock, M., Material form and force. Architectural Design, 82(2), pp. 104-111, 2012. http://dx.doi.org/10.1002/ad.1386

[4] Semper, G., Style in the Technical and Tectonic Arts; or, Practical Aesthetics, Los Angeles: Getty Publications, 2004.

[5] Evans, R., Translations from Drawing to Building and Other Essays, London: Architectural Association, 1997.

[6] Bak, A., Shepherd, P. \& Richens, P., Intuitive interactive form finding of optimised fabric-cast concrete. In Second International Conference on Flexible Formwork (icff2012), University of Bath: Bath, 2012.

[7] Verhaegh, R., Free Forms in Concrete, Eindhoven University of Technology, 2010.

[8] Kotnik, T. \& Weinstock, M., Material, form and force. Architectural Design, 82(2), pp. 104-111, 2012.

[9] Khoshnevis, B., Contour Crafting, University of Southern California, available at: http://www.contourcrafting.org/. (accessed 9 March 2015).

[10] Lim, S., Buswell, R., Le, T., Wackrow, R., Austin, S., Gibb, A. \& Thorpe, T., Development of a viable concrete printing process. Proceedings of the 28th International Symposium on Automation and Robotics in Construction (ISARC2011), pp. 665-670, 2011.

[11] Lloret, E., Shahab, A.R., Linus, M., Flatt, R.J., Gramazio, F., Kohler, M. \& Langenberg, S., Complex concrete structures: merging existing casting techniques with digital fabrication. Computer Aided Design, 60, pp. 40-49, 2015.

http://dx.doi.org/10.1016/j.cad.2014.02.011

[12] Oesterle, S., Vansteenkiste, A. \& Mirjan, A., 24 zero waste free-form formwork, pp. 258-267, 2010.

[13] Sondergaard, A. \& Dombernowsky, P., Unikabeton prototype. In Fabricate: Making Digital Architecture, 2011.

[14] Oxman, N., Keating, S. \& Klein, J., Building-scale 3D printing, available at: https:// www.media.mit.edu/research/groups/mediated-matter (accessed 10 May 2015).

[15] Hack, N., Lauer, W.V., Gramazio, F., Kohler, M. \& Langenberg, S., Mesh-mould: robotically fabricated spatial meshes as concrete formwork and reinforcement. Architecture Design, 84(3), pp. 44-53, 2014. http://dx.doi.org/10.1002/ad.1753

[16] Xu, S., Yan, Z., Jang, K., Huang, W., Fu, H., Kim, J., et al., Assembly of micro/nanomaterials into complex, three-dimensional architectures by compressive buckling. Science, 347(6218), pp. 154-159, 2014. http://dx.doi.org/10.1126/science. 1260960

[17] Verduzco, R., Shape-shifting liquid crystals. Science, 347(6225), pp. 949-950, 2015. http://dx.doi.org/10.1126/science.aaa6579 
[18] Thompson, R., Manufacturing Processes for Design Professionals, New York: Thames \& Hudson, 2007.

[19] Nahmad Vazquez, A., Wintour, P., Leydi, E. \& Sosa-Mejia, R., “Symbiotica," Architectural Association, 2010.

[20] Nahmad Vazquez, A., Bhooshan, S., Sondergaard, A., Inamura, C., Zabel, C. \& El Sayed, M., Design, analysis and fabrication of expressive, efficient shell structures: a prototype exploring synergy between architecture, engineering and manufacture. In Proceedings of the IASS-SLTE 2014 Symposium, International Association for Shell and Spatial Structures: Brazil, 2014.

[21] Bhooshan, S. \& El Sayed, M., Use of sub-division surfaces in architectural form-finding and procedural modelling. In Proceedings of the 2011 Symposium on Simulation for Architecture and Urban Design, pp. 60-67, 2011.

[22] Williams, N., Stehling, H., Scheurer, F., Oesterle, S., Kohler, M. \& Gramazio, F., A case study of a collaborative digital workflow in the design and production of formwork for 'non-standard' concrete structures. International Journal of Architectural Computing, 9(3), pp. 223-240, 2011.

http://dx.doi.org/10.1260/1478-0771.9.3.223

[23] Chua, C.K., Leong, K.F. \& Lim, C.S., Rapid Prototyping: Principles and Applications, World Scientific, 2010. http://dx.doi.org/10.1142/6665

[24] Ye, B.C. \& Tsukruk, V.V., Designing two-dimensional materials that spring rapidly into three-dimensional shapes. Science, 347, pp. 130-131, 2015.

http://dx.doi.org/10.1126/science.aaa2643 